comparison with the group studied in 1998 (13.5\% v. 10\%). Overweight in boys aged 9 years occurred more often in 2008 in comparison with the study of peers in 1998. We did not find significant differences in prevalence of overweight for the remaining analysed boys' age groups. Variation in prevalence of obesity in boys from both the groups analysed were not statistically significant $(7 \cdot 3 \% v \cdot 6 \cdot 2 \%)$. We did not find considerable differences in the prevalence of obesity in boys for individual age groups in 1998 and 2008 studies.
Variation in prevalence of overweight $(12 \cdot 1 \% v .13 \cdot 4 \%)$ and obesity $(10 \cdot 3 \% v \cdot 7 \cdot 8 \%)$ in girls at both the groups analysed were not statistically significant. We did not find substantial differences in prevalence of overweight and obesity in girls for individual age group in the 1998 and 2008 studies.

Conclusions: Our study demonstrates stabilization in girls' overweight and obesity in comparison to a slight increase in boys' overweight in schoolchildren from rural area in South-East of Poland.

\title{
Poster Abstracts: Background to Childhood Obesity
}

\section{3 - Large portions of entrees in school lunches and obesity risk in children living in Piedmont, a Northern Italian region}

\author{
TD Spagnoli ${ }^{1}$, A Costa ${ }^{2}$, M Caputo $^{2}$ and L Cesari ${ }^{1}$
}

${ }^{1}$ ASL TO 3 Service of Nutrition and Food Hygiene, Collegno, Turin, Italy: ${ }^{2}$ Piedmont Regional Department for Health Promotion, Turin, Italy

Introduction: Large entree portion-sizes may be 'obesogenic', producing excessive intake at meals especially in preschool children/schoolchildren with satiety deficits.

Method: In 2007-2009, using a checklist, we assessed in Piedmont (an Italian Region), in a random sample of 1733 schools, whether the procedures for calibrating servings were consistent with the evidence-based systems suggested by literature (calibrated tools or 'sample-dishes'). Foods portioned at canteens, such as pasta (entree in Italy) or vegetables (side-dish) were studied. Foods pre-weighted in the cooking-centres (meat, etc.) were excluded. Some of the sampled elementary schools were evaluated also for the food amount served in each child's plate (we weighed twice the full/empty plate), compared with the recommendations in the menu plan (Student's $t$ test).

Results: Pasta/vegetables are served in $64 \%$ of schools 'a glance', in $16 \%$ by calibrated tools (93\% skimmers have the same diameter for different age groups) and in $14 \%$ by sample dishes. Seventy-nine per cent allow the children to be served twice if they ask. Significant differences between the mean served amounts (pasta $67+7 \mathrm{~g} /$ vegetables $86+34 \mathrm{~g}$ ) and the recommendations (pasta $60+0 \mathrm{~g} /$ vegetables $106+31 \mathrm{~g}$ ) were found $(P<0 \cdot 05)$.

Conclusions: The 'a glance' method preferred by the school canteen staff has a wide inter-operator variability and is not consistent with literature guidelines. The risk is to promote overeating for pasta and a compensatory decrease for vegetables even if the menu plan is nutritionally balanced. Manuals to ensure suitable servings are spread in the USA and included into specific SchoolMeals-Programs, so we are going to promote similar tools in Piedmont too, verifying their applicability through surveillance actions.

\section{4 - Evaluation of dietary quality in relation to body composition and socio-economic status in European adolescents}

\author{
K Vyncke ${ }^{1}$, I Huybrechts ${ }^{1}, M$ Van Winckel ${ }^{2}, M$ Gonzalez Gross ${ }^{3}$, P Stehle ${ }^{3}$, \\ F Gottrand $^{4}$, T Mouratidou ${ }^{5}$ and S De Henauw ${ }^{1}$, on behalf of the HELENA Consortium \\ 'Department of Public Health, Blok A, De Pintelaan 185, Ghent, Belgium: ${ }^{2}$ Ghent University, Ghent, Belgium: \\ ${ }^{3}$ Rheinische Friedrich Wilhelms Universitat, Bonn, Germany: ${ }^{4}$ Universite de Lille, Lille, France: ${ }^{5}$ Universidad de \\ Zaragoza, Zaragoza, Spain
}

